\title{
Perdas Econômicas \\ dos Desastres Climáticos \\ no Estado do Rio de Janeiro, 2001-2010
}

\author{
Carlos Eduardo Frickmann Young \\ Camilla Aguiar \\ Elisa Possas ${ }^{1}$
}

\begin{abstract}
Resumo
Este trabalho estima as perdas econômicas decorrentes de enchentes e movimentos de massa causados por eventos climáticos extremos (chuvas torrenciais) no Estado do Rio de Janeiro no período 2001-2010. Foram utilizados os dados do Atlas Brasileiro de Desastres Naturais referentes ao número de pessoas afetadas, frequência e locais de ocorrência desses eventos. Essas informações foram cruzadas com estimativas de perdas econômicas na Região Serrana do Rio de Janeiro causadas pela tragédia de janeiro de 2011 (Banco Mundial, 2012). Assim, estimou-se que as perdas com desastres semelhantes ocorridos no Estado do Rio de Janeiro no período estudado ficaram entre $R \$ 48,4$ e 54,5 bilhões, aproximadamente 1,3\% do PIB do Estado em 2010.
\end{abstract}

Palavras-chave: Desastres climáticos; Inundações; Deslizamentos; Valoração ambiental; Rio de Janeiro

\begin{abstract}
Abstratc
This work estimates the economic losses caused by floods and landslides from extreme weather events (torrential rains) in the State of Rio de Janeiro in the period 2001-2010. We used data from the National Atlas of Natural Disasters for the number of people affected, frequency and locations of these events. These figures were crossed with estimates of economic losses caused by the tragedy in January 2011 in the mountain region surrounding Rio de Janeiro (World Bank, 2012). It was estimated that similar losses in the state of Rio de Janeiro, during the study period, were between $R \$ 48.4$ and 54.5 billion, approximately 1.3\% of the State GDP in 2010.
\end{abstract} Keywords: Climatic disasters; Floods; Landslides; Environmental Valuation; Rio de Janeiro

\footnotetext{
1 Carlos Eduardo Frickmann Young é PhD, Instituto de Economia, UFRJ. Professor Associado do IE/UFRJ e pesquisador do Instituto Nacional de Ciência e Tecnologia de Políticas Públicas, Estratégias e Desenvolvimento (INCT/PPED). young@ie.ufrj.br 


\section{Introdução}

Há cada vez mais consenso entre os cientistas sobre a elevada intensidade e relevância das mudanças climáticas. Embora os cenários de longo prazo envolvam grande incerteza, pois o período de observação ainda é muito curto, não há mais dúvida de que os efeitos serão múltiplos, pois tudo está relacionado à temperatura, chuva, nível do mar, biodiversidade e outros elementos que serão diretamente afetados pela mudança climática.

Já existe consenso sobre a irreversibilidade do processo, e o máximo que se conseguirá nas próximas décadas é evitar que as mudanças climáticas sejam ainda mais drásticas. Assim, a discussão sobre adaptação e ajuste às mudanças começa a ganhar maior peso, já que antes o foco estava quase inteiramente voltado para a questão de como evitar as emissões. Como o Brasil está situado em partes relativamente quentes do planeta e possui dimensões continentais, os impactos serão consideráveis.

Uma das consequências mais dramáticas previstas em função dessas mudanças climáticas associadas ao contínuo incremento na concentração de gases de efeito estufa é o aumento da frequência de eventos climáticos extremos. Embora não exista ainda suficiente evidência empírica para afirmar que, indubitavelmente, o aumento recente na frequência desses fenômenos já se deve às mudanças climáticas, percebe-se que, de qualquer modo, o número de eventos e a seriedade das perdas decorrentes é cada vez maior.

No caso do Estado do Rio de Janeiro, a consequência mais importante desses fenômenos é o aumento na ocorrência de chuvas torrenciais com fortes impactos para a população. As perdas associadas a esses desastres naturais manifestam-se em várias dimensões, sendo que as de maior impacto direto são as perdas de vidas humanas e o deslocamento de populações afetadas, cujo reassentamento pode demorar bastante tempo. Porém também existem consequências econômicas importantes, que incluem a paralisação ou desestabilização de atividades produtivas nos municípios afetados e a necessidade de gastos públicos, tanto de caráter emergencial quanto de reconstrução nas áreas atingidas.

O presente trabalho concentra-se na avaliação dessas perdas econômicas, buscando estimar perdas na produção em função de dias de trabalho suspensos e danos de infraestrutura e outras formas de capital. Os resultados alcançados foram significativos, com perdas e danos estimados para o período 2001-2010 no Estado do Rio de Janeiro entre R\$ 48,4 e 54,5 bilhões, algo em torno do valor médio anual de 1,3\% do PIB do Estado em 2010. A distribuição desses impactos não é uniforme, e alguns municípios acabam sofrendo muito mais do que a média - existe forte concentração dos problemas em um conjunto relativamente reduzido de municípios (19 municípios, de um total de 92, registraram metade dos desastres climáticos no período 2001-2010), embora não se possa apontar para uma maior concentração em áreas específicas do Estado.

Infelizmente, as administrações públicas ainda estão longe de perceber a seriedade da questão, e não há formulação de políticas públicas ou dotação de recursos técnicos e orçamentários destinados a ações preventivas em volume proporcional à relevância do tema. Dada a magnitude das perdas já observadas, que deverão crescer no futuro devido à irreversibilidade das mudanças climáticas, é fundamental que o tema passe a ganhar prioridade entre os tomadores de decisão, bem como na população como um todo. 


\section{Desastres naturais no Rio de Janeiro}

Segundo o Atlas Brasileiro de Desastres Naturais (CEPED, 2011a, 2011b), 89\% dos desastres registrados no Estado do Rio de Janeiro entre 2001 e 2010 foram diretamente relacionados com a ocorrência de fortes precipitações, a saber, inundações bruscas ou graduais, e movimentos de massa. Considerando-se toda a série disponibilizada pelo estudo (19912010), foram ao todo 557 ocorrências de tais desastres, que tiveram como consequência um total de 422.036 desabrigados e desalojados, 1.782 mortos e 3.715 .656 afetados.

Os dados sobre mortos e feridos também são dramáticos. Segundo CEPED (2011b), entre 1991 e 2010, 1287 pessoas haviam morrido por deslizamentos de massa, 300 por inundações bruscas e 195 por inundações graduais. Somando-se esses, contou-se mais de 12 mil feridos e 7 mil pessoas enfermas no período.

Deve-se ressaltar que esses números consideram múltipla contagem (uma mesma pessoa pode ter sido afetada em mais de uma ocasião) mas, ainda sim, demonstram a gravidade desses desastres para a sociedade fluminense: caso esse número de pessoas atingidas seja dividido pela população do Estado do Rio de Janeiro (16,0 milhões), o resultado é uma relação de $23,2 \%$. Ou seja, em média para os vinte anos considerados pela série, mais de $1 \%$ da população do estado é afetada anualmente por desastres causados por eventos climáticos extremos.

Ainda mais grave é o fato de que mais de $90 \%$ dos registros (520 de 557) concentraramse na segunda metade do período analisado (2001-2010). Isso demonstra uma preocupante tendência de crescimento na quantidade dessas ocorrências (Figura 1 e Tabela 1).

FIGURA 1

Registros de desastres no Estado do Rio de Janeiro

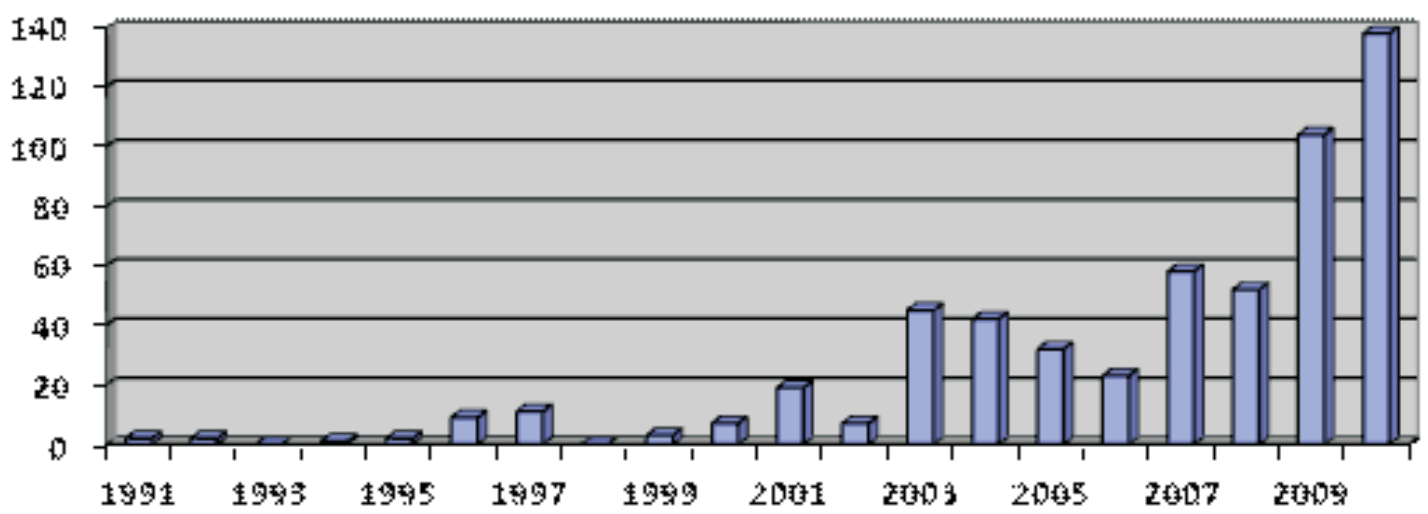

Fonte: Elaboração própria a partir do Atlas Brasileiro de Desastres Naturais (CEPED, 2011b).

Tabela 1

Estimativa de danos humanos, 1991-2010, Estado do Rio de Janeiro

\begin{tabular}{|c|c|c|c|c|}
\hline & $1991-2010$ & $2001-2010$ & 520 \\
\hline Total de registros & 37 & 3.468 .835 & 3.715 .656 \\
\hline Afetados & 246.821 & 69.532 & 74.480 \\
\hline Desabrigados & 4.948 & 324.469 & 347.556 \\
\hline Desalojados & 23.087 & \\
\hline
\end{tabular}


Existem várias possíveis explicações para esse acelerado aumento em número de eventos: - Melhoria na cobertura das informações (ou seja, no passado havia mais desastres que deixavam de ser registrados oficialmente pela Defesa Civil);

- Aumento da densidade populacional nas áreas de risco (ou seja, mais pessoas passaram a morar em áreas onde os fenômenos estudados ocorrem);

- Aumento na frequência de chuvas torrenciais e outros fenômenos climáticos extremos já são consequência das mudanças climáticas previstas pela grande maioria da comunidade científica.

O mais provável é que todas essas hipóteses estejam corretas, e que existe uma combinação perversa entre o aumento da população vivendo em áreas de risco e a maior probabilidade de ocorrência de eventos climáticos extremos. Assim, observa-se não só a relevância que os impactos de tais acontecimentos têm no presente, mas também o fato de que, caso não ocorram avanços significativos na sua prevenção, as consequências negativas só aumentarão, o que torna essencial a formulação de políticas públicas para lidar com o tema.

\section{Metodologia}

Pelas razões descritas na seção anterior, este trabalho concentrou-se na estimativa das perdas e danos econômicos causados por desastres no Estado do Rio de Janeiro no período 2001-2010. No entanto, é importante ressaltar que nem todos os dados necessários para o exercício estão disponíveis, tais como a intensidade e o número de pessoas atingidas por evento (os dados disponíveis referem-se ao número de eventos e população atingida por ano, mas não identifica a distribuição entre eles). Por isso, uma proxy foi elaborada para estimar o impacto dos eventos por município, considerando que a população atingida distribui-se proporcionalmente ao número de registros de desastres no município naquele ano.

Não existem estimativas sistemáticas acerca das perdas econômicas associadas a esses desastres. Mas uma importante contribuição foi o relatório feito pelo Banco Mundial (2012) contendo uma detalhada avaliação dos efeitos das fortes chuvas que atingiram a Região Serrana do estado em 11 e 12 de janeiro de 2011.

Nesse evento, chuvas torrenciais ocasionaram enchentes e deslizamentos de terra em diversos municípios, sendo os mais afetados Nova Friburgo (onde choveu $166 \mathrm{~mm}$ nesses dois dias, o que representa mais de 70\% do valor médio histórico para o mês de janeiro), Teresópolis, Petrópolis, Sumidouro, São José do Vale do Rio Preto, Bom Jardim e Areal. Estima-se que mais de 900 pessoas morreram, e mais de 300 mil foram afetadas (Banco Mundial, 2012).

O estudo inclui uma extensa estimativa de perdas e danos, diretos e indiretos, além de dados relativos aos danos humanos. As perdas e danos foram calculados a partir de dados oficiais, considerando-se quatro setores:

- infraestrutura (subdividida

- em transporte, telecomunicações, água e saneamento e energia),

- setores sociais (subdivididos em habitação, saúde e educação),

- setores produtivos (subdivididos em agricultura, indústria, comércio e turismo) e

- meio ambiente.

No entanto, o relatório chama atenção para o fato de que alguns desses subsetores, principalmente saúde e educação, não dispunham de informações detalhadas sobre suas respectivas perdas e danos. Portanto, vários custos acabaram omitidos do cálculo final que, por 
isso, deve ser entendido como uma subestimativa da perda total, ainda mais se lembrarmos que aspectos imateriais, como desconforto e outras formas de perda de qualidade de vida, não foram valorados, bem como as perdas de vidas humanas.

Embora o evento referido esteja fora do período coberto pelo Atlas de Desastres Naturais e o valor da perda subestimado, as estimativas desses danos permitiram calcular coeficientes médios em termos de danos per capita por pessoa afetada, desabrigada ou desalojada, que foram utilizados para avaliar as perdas econômicas no período 2001-2010.

Na avaliação do Banco Mundial, o total de perdas e danos na tragédia de janeiro de 2011 foi estimado em $\mathrm{R} \$ 4.785$ milhões de reais (a preços de 2010). Os danos humanos também atingiram números excepcionalmente elevados, incluindo 38.937 desabrigados e desalojados, e 304.562 afetados. Com base nesses dados, foram estimados os seguintes coeficientes médios de dano per capita: R\$ 15.713,97 por pessoa afetada, R\$ 290.793,44 por desabrigado, $\mathrm{R} \$ 212.904,42$ por desalojado, e R\$122.913,38 por pessoa desabrigada ou desalojada (Tabela 2).

Tabela 2

Estimativa de coeficientes de dano per capita a partir da tragédia de Janeiro de 2011 na Serra Fluminense

\begin{tabular}{c|c}
\hline Total de Perdas e Danos & R\$ $4.785 .878 .382,00$ \\
\hline Total de Pessoas Afetadas & 304.562 \\
\hline Total de Desabrigados & 16.458 \\
\hline Total de Desalojados & 22.479 \\
\hline$R \$ /$ Afetado & $\mathrm{R} \$ 15.713,97$ \\
\hline $\mathrm{R} \$$ Desabrigado & $\mathrm{R} \$ 290.793,44$ \\
\hline $\mathrm{R} \$ /$ Desalojado & $\mathrm{R} \$ 212.904,42$ \\
\hline $\mathrm{R} \$$ Desabrigado+Desalojado & $\mathrm{R} \$ 122.913,38$ \\
\hline
\end{tabular}

Fonte: Elaboração própria, baseado em Banco Mundial 2012.

Cada um desses coeficientes foi, então, multiplicado pelo respectivo número de casos registrados no Estado do Rio de Janeiro acumulados na década 2001/2010, e depois dividido por dez para alcançar o valor médio anual. Dessa forma, as estimativas de perdas econômicas médias anuais associadas a esses eventos extremos foram as seguintes, com os resultados apresentados na Tabela 3. O maior valor, $\mathrm{R} \$ 69,1$ bilhões, foi alcançado usando o coeficiente de perdas por pessoas afetadas, e o menor valor foi $\mathrm{R} \$ 20,2$ bilhões, considerando o coeficiente de perdas por pessoas desabrigadas. É interessante notar que os resultados situam-se em ordens de grandeza próxima (dezenas de bilhões de reais), e o valor médio dessas estimativas é de $\mathrm{R} \$ 46,5$ bilhões (ou R $\$ 4,5$ bilhões por ano). Em termos relativos, esses valores oscilam entre $0,5 \%$ e 1,6\% do PIB fluminense de 2010, com média em 1,1\%.

Tabela 3

Estimativa de perdas econômicas no Estado do Rio de Janeiro causadas por inundações e deslizamentos de terra, 2001/2010

\begin{tabular}{|c|c}
\hline Total de Afetados & 3.468 .835 \\
\hline Total de Desabrigados & 69.532 \\
\hline Total de Desalojados & 324.469 \\
\hline Perda por Afetados (média anual) & $\mathrm{R} \$ 54.509 .171 .982,14$ \\
\hline Perda por Desabrigados (média anual) & $\mathrm{R} \$ 20.219 .449 .243,97$ \\
\hline Perda por Desalojados (média anual) & $\mathrm{R} \$ 69.080 .883 .167,81$ \\
\hline Perda por Desabrigados + Desalojados (média anual) & $\mathrm{R} \$ 48.427 .995 .695,26$
\end{tabular}


Embora esses valores sejam significativamente altos, deve-se notar que as perdas para anos mais recentes estão subestimadas, visto que o número de eventos climáticos extremos vem crescendo com o tempo, e o cálculo foi efetuado com base no valor médio para a década. Outra subestimativa importante é o não tratamento das informações sobre mortos e feridos: nenhuma consideração de valoração dessas perdas foi efetuada neste exercício, bem como perdas materiais de qualidade de vida (desconforto, insegurança e perdas afetivas). Ainda assim, demonstra-se que o volume anual de perdas com desastres naturas atinge valores extremamente altos e, por isso, políticas de prevenção e adaptação deveriam ser prioritárias.

A distribuição dos desastres não é homogênea pelos 92 municípios fluminenses (Tabela 4). Desse total, 19 municípios concentraram 50,6\% do número de ocorrências registradas, apesar de somarem 26,3\% do PIB Estadual e 26,2\% da população. Esses municípios são Petrópolis, São Gonçalo, Angra do Reis, Bom Jesus do Itabapoana, Belfort Roxo, Campos dos Goytacazes, Guapimirim, Mangaratiba, Teresópolis, Duque de Caxias, Natividade, Silva Jardim, Barra Mansa, Macaé, Rio Bonito, Aperibé, Itaperuna, Paraíba do Sul e Piraí.

Tabela 4

Percentual de número de eventos (inundações e deslizamentos de terra) e características socioeconômicas dos municípios fluminenses, $2001 / 2010$

\begin{tabular}{|c|c|c|c|c|c|c|}
\hline Município & $\begin{array}{r}\text { Total de } \\
\text { Desastres }\end{array}$ & População (2010) & $\begin{array}{c}\text { Participação na } \\
\text { população estadual }\end{array}$ & $\begin{array}{l}\text { PIB } 2010 \text { a Preço } \\
\text { Correntes (R\$1000) }\end{array}$ & $\begin{array}{l}\text { Participação no } \\
\text { PIB estadual }\end{array}$ & $\begin{array}{c}\% \text { dos } \\
\text { desastres }\end{array}$ \\
\hline Petrópolis & 28 & 296.044 & $1,9 \%$ & 7.063.116 & $1,7 \%$ & $5,4 \%$ \\
\hline São Gonçalo & 27 & 999.901 & $6,3 \%$ & 10.340 .756 & $2,5 \%$ & $5,2 \%$ \\
\hline Angra do Reis & 19 & 169.270 & $1,1 \%$ & 10.176 .448 & $2,5 \%$ & $3,7 \%$ \\
\hline Bom Jesus do Itabapoana & 19 & 35.384 & $0,2 \%$ & 406.723 & $0,1 \%$ & $3,7 \%$ \\
\hline Belfort Roxo & 16 & 469.261 & $2,9 \%$ & 4.467 .555 & $1,1 \%$ & $3,1 \%$ \\
\hline Campos dos Goytacazes & 16 & 463.545 & $2,9 \%$ & 25.313.179 & $6,2 \%$ & $3,1 \%$ \\
\hline Guapimirim & 15 & 51.487 & $0,3 \%$ & 485.269 & $0,1 \%$ & $2,9 \%$ \\
\hline Mangaratiba & 12 & 36.311 & $0,2 \%$ & 939.819 & $0,2 \%$ & $2,3 \%$ \\
\hline Teresópolis & 12 & 163.805 & $1,0 \%$ & 2.764 .129 & $0,7 \%$ & $2,3 \%$ \\
\hline Duque de Caxias & 11 & 855.046 & $5,3 \%$ & 26.496 .845 & $6,5 \%$ & $2,1 \%$ \\
\hline Natividade & 11 & 15.077 & $0,1 \%$ & 154.096 & $0,0 \%$ & $2,1 \%$ \\
\hline Silva Jardim & 11 & 21.360 & $0,1 \%$ & 192.515 & $0,0 \%$ & $2,1 \%$ \\
\hline Barra Mansa & 10 & 177.861 & $1,1 \%$ & 3.205 .477 & $0,8 \%$ & $1,9 \%$ \\
\hline Macaé & 10 & 206.748 & $1,3 \%$ & 11.267 .976 & $2,8 \%$ & $1,9 \%$ \\
\hline Rio Bonito & 10 & 55.586 & $0,3 \%$ & 856.989 & $0,2 \%$ & $1,9 \%$ \\
\hline Aperibé & 9 & 10.215 & $0,1 \%$ & 91.384 & $0,0 \%$ & $1,7 \%$ \\
\hline Itaperuna & 9 & 95.876 & $0,6 \%$ & 1.408 .393 & $0,3 \%$ & $1,7 \%$ \\
\hline Paraíba do Sul & 9 & 41.088 & $0,3 \%$ & 532.077 & $0,1 \%$ & $1,7 \%$ \\
\hline Piraí & 9 & 26.309 & $0,2 \%$ & 1.046 .509 & $0,3 \%$ & $1,7 \%$ \\
\hline Barra do Piraí & 8 & 94.855 & $0,6 \%$ & 1.386 .837 & $0,3 \%$ & $1,5 \%$ \\
\hline Cambuci & 8 & 14.829 & $0,1 \%$ & 160.292 & $0,0 \%$ & $1,5 \%$ \\
\hline Macuco & 8 & 5.269 & $0,0 \%$ & 82.999 & $0,0 \%$ & $1,5 \%$ \\
\hline Miracema & 8 & 26.829 & $0,2 \%$ & 257.331 & $0,1 \%$ & $1,5 \%$ \\
\hline Cardoso Moreira & 7 & 12.540 & $0,1 \%$ & 119.598 & $0,0 \%$ & $1,3 \%$ \\
\hline Italva & 7 & 14.027 & $0,1 \%$ & 134.321 & $0,0 \%$ & $1,3 \%$ \\
\hline Magé & 7 & 228.150 & $1,4 \%$ & 2.065 .537 & $0,5 \%$ & $1,3 \%$ \\
\hline Mendes & 7 & 17.940 & $0,1 \%$ & 179.644 & $0,0 \%$ & $1,3 \%$ \\
\hline Niterói & 7 & 487.327 & $3,0 \%$ & 11.214 .103 & $2,8 \%$ & $1,3 \%$ \\
\hline
\end{tabular}




\begin{tabular}{|c|c|c|c|c|c|c|}
\hline Paty dos Alferes & 7 & 26.381 & $0,2 \%$ & 274.002 & $0,1 \%$ & $1,3 \%$ \\
\hline Laje do Muriaé & 6 & 7.491 & $0,0 \%$ & 76.962 & $0,0 \%$ & $1,2 \%$ \\
\hline Porciúncula & 6 & 17.771 & $0,1 \%$ & 188.163 & $0,0 \%$ & $1,2 \%$ \\
\hline Valença & 6 & 71.894 & $0,4 \%$ & 986.897 & $0,2 \%$ & $1,2 \%$ \\
\hline Comendador Levy Gasparian & 5 & 8.183 & $0,1 \%$ & 127.894 & $0,0 \%$ & $1,0 \%$ \\
\hline Itaboraí & 5 & 218.090 & $1,4 \%$ & 2.186 .487 & $0,5 \%$ & $1,0 \%$ \\
\hline Japeri & 5 & 95.391 & $0,6 \%$ & 916.884 & $0,2 \%$ & $1,0 \%$ \\
\hline Paraty & 5 & 37.575 & $0,2 \%$ & 632.802 & $0,2 \%$ & $1,0 \%$ \\
\hline Queimados & 5 & 137.938 & $0,9 \%$ & 1.670 .541 & $0,4 \%$ & $1,0 \%$ \\
\hline Santa Maria Madalena & 5 & 10.321 & $0,1 \%$ & 124.523 & $0,0 \%$ & $1,0 \%$ \\
\hline Santo Antônio de Pádua & 5 & 40.569 & $0,3 \%$ & 554.009 & $0,1 \%$ & $1,0 \%$ \\
\hline São Fidélis & 5 & 37.553 & $0,2 \%$ & 427.819 & $0,1 \%$ & $1,0 \%$ \\
\hline São Francisco de Itabapoana & 5 & 41.357 & $0,3 \%$ & 616.362 & $0,2 \%$ & $1,0 \%$ \\
\hline São João de Meriti & 5 & 459.356 & $2,9 \%$ & 4.826 .212 & $1,2 \%$ & $1,0 \%$ \\
\hline Trajano de Moraes & 5 & 10.281 & $0,1 \%$ & 91.625 & $0,0 \%$ & $1,0 \%$ \\
\hline Cachoeiras de Macacu & 4 & 54.370 & $0,3 \%$ & 923.618 & $0,2 \%$ & $0,8 \%$ \\
\hline Casimiro de Abreu & 4 & 35.373 & $0,2 \%$ & 1.716 .204 & $0,4 \%$ & $0,8 \%$ \\
\hline Mesquita & 4 & 168.403 & $1,1 \%$ & 1.533 .364 & $0,4 \%$ & $0,8 \%$ \\
\hline Nova Friburgo & 4 & 182.016 & $1,1 \%$ & 2.835 .809 & $0,7 \%$ & $0,8 \%$ \\
\hline Nova Iguaçu & 4 & 795.212 & $5,0 \%$ & 9.496 .660 & $2,3 \%$ & $0,8 \%$ \\
\hline Paracambi & 4 & 47.074 & $0,3 \%$ & 498.103 & $0,1 \%$ & $0,8 \%$ \\
\hline Rio Claro & 4 & 17.401 & $0,1 \%$ & 198.520 & $0,0 \%$ & $0,8 \%$ \\
\hline São João da Barra & 4 & 32.767 & $0,2 \%$ & 3.484 .707 & $0,9 \%$ & $0,8 \%$ \\
\hline São Pedro da Aldeia & 4 & 88.013 & $0,6 \%$ & 940.787 & $0,2 \%$ & $0,8 \%$ \\
\hline Sapucaia & 4 & 17.504 & $0,1 \%$ & 403.509 & $0,1 \%$ & $0,8 \%$ \\
\hline Saquarema & 4 & 74.221 & $0,5 \%$ & 946.030 & $0,2 \%$ & $0,8 \%$ \\
\hline Seropédica & 4 & 78.183 & $0,5 \%$ & 852.538 & $0,2 \%$ & $0,8 \%$ \\
\hline Tanguá & 4 & 30.731 & $0,2 \%$ & 290.655 & $0,1 \%$ & $0,8 \%$ \\
\hline Três Rios & 4 & 77.503 & $0,5 \%$ & 1.733 .224 & $0,4 \%$ & $0,8 \%$ \\
\hline Cantagalo & 3 & 19.826 & $0,1 \%$ & 563.461 & $0,1 \%$ & $0,6 \%$ \\
\hline Conceição de Macabu & 3 & 21.200 & $0,1 \%$ & 185.020 & $0,0 \%$ & $0,6 \%$ \\
\hline Itaocara & 3 & 22.902 & $0,1 \%$ & 258.102 & $0,1 \%$ & $0,6 \%$ \\
\hline Nilópolis & 3 & 157.483 & $1,0 \%$ & 1.668 .960 & $0,4 \%$ & $0,6 \%$ \\
\hline Resende & 3 & 119.801 & $0,7 \%$ & 6.417 .157 & $1,6 \%$ & $0,6 \%$ \\
\hline Rio de Janeiro & 3 & 6.323 .037 & $39,5 \%$ & 190.249 .043 & $46,7 \%$ & $0,6 \%$ \\
\hline Sumidouro & 3 & 14.920 & $0,1 \%$ & 192.095 & $0,0 \%$ & $0,6 \%$ \\
\hline Varre-Sai & 3 & 9.503 & $0,1 \%$ & 95.982 & $0,0 \%$ & $0,6 \%$ \\
\hline Araruama & 2 & 112.028 & $0,7 \%$ & 1.264 .898 & $0,3 \%$ & $0,4 \%$ \\
\hline Areal & 2 & 11.421 & $0,1 \%$ & 195.925 & $0,0 \%$ & $0,4 \%$ \\
\hline Carapebus & 2 & 13.348 & $0,1 \%$ & 1.119 .357 & $0,3 \%$ & $0,4 \%$ \\
\hline Carmo & 2 & 17.439 & $0,1 \%$ & 392.686 & $0,1 \%$ & $0,4 \%$ \\
\hline Cordeiro & 2 & 20.403 & $0,1 \%$ & 210.956 & $0,1 \%$ & $0,4 \%$ \\
\hline Engenheiro Paulo de Frontin & 2 & 13.239 & $0,1 \%$ & 147.459 & $0,0 \%$ & $0,4 \%$ \\
\hline Iguaba Grande & 2 & 22.858 & $0,1 \%$ & 236.044 & $0,1 \%$ & $0,4 \%$ \\
\hline Maricá & 2 & 127.519 & $0,8 \%$ & 1.644 .995 & $0,4 \%$ & $0,4 \%$ \\
\hline Quatis & 2 & 12.831 & $0,1 \%$ & 186.195 & $0,0 \%$ & $0,4 \%$ \\
\hline Quissamã & 2 & 20.244 & $0,1 \%$ & 3.112 .919 & $0,8 \%$ & $0,4 \%$ \\
\hline Rio das Flores & 2 & 8.545 & $0,1 \%$ & 332.663 & $0,1 \%$ & $0,4 \%$ \\
\hline
\end{tabular}




\begin{tabular}{|c|c|c|c|c|c|c|}
\hline São José de Ubá & 2 & 7.003 & $0,0 \%$ & 73.778 & $0,0 \%$ & $0,4 \%$ \\
\hline Bom Jardim & 1 & 25.398 & $0,2 \%$ & 356.335 & $0,1 \%$ & $0,2 \%$ \\
\hline Duas Barras & 1 & 10.933 & $0,1 \%$ & 159.306 & $0,0 \%$ & $0,2 \%$ \\
\hline Itatiaia & 1 & 28.852 & $0,2 \%$ & 1.409 .576 & $0,3 \%$ & $0,2 \%$ \\
\hline Miguel Pereira & 1 & 24.647 & $0,2 \%$ & 330.990 & $0,1 \%$ & $0,2 \%$ \\
\hline Pinheiral & 1 & 22.724 & $0,1 \%$ & 207.797 & $0,1 \%$ & $0,2 \%$ \\
\hline São José do Vale do Rio Preto & 1 & 20.252 & $0,1 \%$ & 646.954 & $0,2 \%$ & $0,2 \%$ \\
\hline Armação dos Búzios & 0 & 27.538 & $0,2 \%$ & 1.288 .962 & $0,3 \%$ & $0,0 \%$ \\
\hline Arraial do Cabo & 0 & 27.770 & $0,2 \%$ & 357.181 & $0,1 \%$ & $0,0 \%$ \\
\hline Cabo Frio & 0 & 186.222 & $1,2 \%$ & 6.551 .707 & $1,6 \%$ & $0,0 \%$ \\
\hline Itaguaí & 0 & 109.163 & $0,7 \%$ & 4.284 .959 & $1,1 \%$ & $0,0 \%$ \\
\hline Porto Real & 0 & 16.574 & $0,1 \%$ & 4.820 .284 & $1,2 \%$ & $0,0 \%$ \\
\hline Rio das Ostras & 0 & 105.757 & $0,7 \%$ & 6.121 .512 & $1,5 \%$ & $0,0 \%$ \\
\hline São Sebastião do Alto & 0 & 8.906 & $0,1 \%$ & 99.083 & $0,0 \%$ & $0,0 \%$ \\
\hline Vassouras & 0 & 34.439 & $0,2 \%$ & 404.705 & $0,1 \%$ & $0,0 \%$ \\
\hline Volta Redonda & 0 & 257.996 & $1,6 \%$ & 9.170 .922 & $2,3 \%$ & $0,0 \%$ \\
\hline Rio de Janeiro & 520 & 15.993 .583 & $100,0 \%$ & 407.122 .794 & $100,0 \%$ & $100,0 \%$ \\
\hline
\end{tabular}

Fonte: Elaboração própria a partir das tabelas anteriores e IBGE

Ou seja, existe um conjunto de municípios mais afetados que tem sua população e economia fortemente prejudicadas, e vários deles possuem capacidade bastante limitada de resposta a esses desastres. É importante ressaltar que, no subconjunto dos municípios onde a frequência de desastres naturais ficou bastante acima da média, sua distribuição foi dividida em diversas sub-regiões do Estado do Rio de Janeiro: Serra Fluminense (Petrópolis), Baixada Fluminense (São Gonçalo, Belfort Roxo), Sul (Angra dos Reis) e Norte (Bom Jesus do Itabapoana, Campos dos Goytacazes). Isso indica que o problema se dá ao longo de todo o Estado, e não concentrado apenas nas áreas mais montanhosas.

Um exercício simples permite verificar o efeito devastador dessas perdas na economia municipal. Na cidade de Petrópolis, a mais afetada por desastres no período estudado, enquanto o PIB municipal representou 1,7\% do PIB total do estado no ano de 2010, os registros de desastres corresponderam a aproximadamente 5,4\% dos desastres na década 2001-2010. Partindo da premissa de que as perdas e danos se dividem igualmente entre ocorrências, pode-se estimar para o ano de 2010, um gasto de R 255 milhões, valor que corresponde a 3,6\% de seu PIB nesse ano. Mais uma vez deve-se ressaltar que essas estimativas não consideram as perdas com a tragédia de janeiro de 2011, quando Petrópolis foi um dos municípios mais afetados. Portanto, não cabe dúvida de que as perdas patrimoniais nesse município afetam profundamente a vida econômica de seus habitantes.

Uma outra característica importante é a ausência de correlação estatística entre a frequência de desastres e o nível de desenvolvimento. A Tabela 5 compara dados de frequência de desastres naturais e o índice de desenvolvimento humano municipal (IDHM) e renda per capita, e fica claro que não se trata de um problema característico por nível de desenvolvimento. Por isso, não se pode esperar que os problemas sejam minimizados com crescimento econômico. Por outro lado, é possível que as áreas mais afetadas sejam depreciadas e acabem concentrando as famílias de renda mais baixa do município. A relação entre nível de desenvolvimento, padrões de renda e o perfil das vítimas de desastres naturais deve ser examinada com mais detalhe em pesquisas futuras. 
Tabela 5

PIB per capita e índice de desenvolvimento humano municipal (IDHM) para os 12 municípios fluminenses mais afetados por desastres

\begin{tabular}{|c|c|c|c|c|}
\hline Município & $\begin{array}{l}\text { Ocorrências } \\
\text { de desastres } \\
(2001-2010)\end{array}$ & PIB per capita 2010 & IDHM 2010 & $\begin{array}{l}\text { Posição no ranking } \\
\text { estadual de IDHM }\end{array}$ \\
\hline Petrópolis & 28 & $23.858,53$ & 0,804 & $7^{0}$ \\
\hline São Gonçalo & 27 & $10.341,78$ & 0,782 & $23^{\circ}$ \\
\hline Angra dos Reis & 19 & $60.119,62$ & 0,772 & $36^{\circ}$ \\
\hline Bom Jesus do Itabapoana & 19 & $11.494,55$ & 0,746 & $56^{\circ}$ \\
\hline Belfort Roxo & 16 & $9.520,41$ & 0,742 & $60^{\circ}$ \\
\hline Campos dos Goytacazes & 16 & $54.607,81$ & 0,752 & $54^{\circ}$ \\
\hline Guapimirim & 15 & $9.425,08$ & 0,739 & $63^{\circ}$ \\
\hline Mangaratiba & 12 & $25.882,49$ & 0,79 & $15^{0}$ \\
\hline Teresópolis & 12 & $16.874,51$ & 0,79 & $16^{0}$ \\
\hline Duque de Caxias & 11 & $30.988,79$ & 0,753 & $52^{\circ}$ \\
\hline Natividade & 11 & $10.220,60$ & 0,736 & $68^{\circ}$ \\
\hline
\end{tabular}

Fonte: Elaboração própria a partir das tabelas anteriores, IBGE e Programa das Nações Unidas para o Desenvolvimento (PNUD)

Em contraste, percebe-se que o tema está longe de receber, por parte das administrações públicas, atenção proporcional à sua importância. A falta de preocupação das administrações públicas fica evidente quando se examinam os dados orçamentários, e o caso capital do Estado, a cidade do Rio de Janeiro, exemplifica isso. Segundo o Fórum do Orçamento Popular (Jornal dos Economistas, abril de 2013), no período 2008-2012, foram executadas somente $62 \%$ das verbas orçamentárias destinadas à prevenção de tais desastres através de ações de estabilização geotécnica, que consistem em obras preventivas em encostas e áreas de risco, com o objetivo de garantir a segurança da população (Tabela 6). Percebe-se que a execução da verba destinada a ações preventivas fica, quase sempre, bastante abaixo da meta. Mesmo no fatídico ano de 2011, marcado pelas chuvas torrenciais de janeiro, apenas $50 \%$ das verbas orçadas foram efetivamente executadas nesse tipo de ação.

A situação em Petrópolis tampouco deixou de ser crítica. Chuvas torrenciais em março de 2013 causaram deslizamentos, em áreas conhecidamente de risco, resultando em 33 mortes. A cidade ainda tem mais de 11 mil residências (12\% do total) localizadas em áreas de alto risco.

Tabela 6

Recursos orçados e executados em ações de estabilização geotécnica, Município do Rio de Janeiro 2008-2013

\begin{tabular}{|c|c|c|c|c|c|}
\hline \multicolumn{6}{|c|}{ Estabilização Geotécnica } \\
\hline Exercício & Autorização inicial (a) & Autorização final (b) & $\begin{array}{r}\text { Variação real da autorização } \\
\text { (b-a) }\end{array}$ & Executado (c) & Taxa de execução (c/b) \\
\hline 2008 & 6.597 .000 & 4.802 .677 & -1.794 .323 & 3.5663652 & $74 \%$ \\
\hline 2009 & 4.518 .117 & 8.626 .726 & 4.108 .609 & 5.879 .147 & $68 \%$ \\
\hline 2010 & 5.283 .526 & 140.430 .728 & 135.147 .202 & 138.418 .186 & $99 \%$ \\
\hline 2011 & 63.148 .552 & 95.001 .433 & 31.852 .881 & 47.190 .145 & $50 \%$ \\
\hline 2012 & 77.571 .438 & 132.614 .788 & 55.043 .350 & 91.337 .511 & $69 \%$ \\
\hline 2013 & 83.939 .611 & 83.939 .611 & - & - & - \\
\hline Total & 240.058 .244 & 465.415 .962 & 224.357.718 & 286.391.641 & $62 \%$ \\
\hline
\end{tabular}

O exemplo acima refere-se ao governo municipal com maior capacidade de lidar com questões de desastres naturais, visto que a cidade do Rio de Janeiro dispõe de muito mais 
recursos humanos, técnicos e financeiros do que seus vizinhos. A situação dos demais municípios fluminenses é seguramente pior, e o agravamento das mudanças climáticas, combinada com a falta de prioridade da questão de prevenção de desastres naturais na agenda de políticas públicas, aponta para um risco crescente de possibilidade de tragédias futuras.

\section{Conclusão}

Este trabalho demonstrou que, se por um lado observamos que as chuvas torrenciais que ocorrem regularmente no Estado no Rio de Janeiro são eventos extremos de causas naturais, sua frequência tende fortemente ao crescimento junto com as mudanças climáticas globais. Como consequência, os custos sociais e econômicos associados a tais eventos deverão também se tornar mais graves mas, ainda assim, há poucas medidas preventivas efetivamente sendo postas em prática.

O altíssimo custo da ocorrência não mitigada desses eventos prejudica a economia e o crescimento dos municípios e do estado enquanto a própria verba destinada a essa prevenção não é devidamente utilizada. A falta de correlação entre incidência e subdesenvolvimento indica que isso não é resultado da impossibilidade de arcar com os gastos de tais políticas, mas sim de falta de interesse ou prioridade de políticas públicas, que vem tendo consequências cada vez maiores para a sociedade.

Por isso, uma preparação para os próximos anos envolve primeiramente uma mudança de postura em relação à forma de lidar com esses eventos, não só através do estímulo a políticas efetivas de prevenção e mitigação de danos, mas também através do monitoramento de risco eficiente. Incorporar a adaptação aos fenômenos climáticos extremos e prover sistemas de defesa civil são passos importantes, bem como a realocação de populações estabelecidas em áreas de risco e outras medidas antecipatórias, são itens que devem constar na pauta de prioridades das administrações públicas em todos os seus níveis.

\section{Referências bibliográficas}

BANCO MUNDIAL. Avaliação de Perdas e Danos: Inundações e Deslizamentos na Região Serrana do Rio de Janeiro. jan. 2011. Relatório elaborado pelo Banco Mundial com apoio do Governo do Estado do Rio de Janeiro. nov. 2012.

CEPED - Centro Universitário de Estudos e Pesquisas sobre Desastres. Atlas brasileiro de desastres naturais 1991 a 2010: volume Brasil. Florianópolis: CEPED UFSC, 2011a. Disponível em: http://www.ceped.ufsc.br/. Acesso em: 26 maio 2013.

. Atlas brasileiro de desastres naturais 1991 a 2010: volume Rio de Janeiro. Florianópolis: CEPED UFSC, 2011b. Disponível em: http://www.ceped.ufsc.br/. Acesso em: 26 maio. 2013.

. Diretrizes em redução de riscos de desastres: região serrana do Rio de Janeiro. Florianópolis: CEPED UFSC, 2011c. Disponível em: http://www.ceped.ufsc.br/. Acesso em: 27 maio 2013.

FÓRUM POPULAR DO ORÇAMENTO - RJ. Todo ano a chuva tem data marcada para pegar o 
governo de surpresa. Jornal dos Economistas, pp. 14-15, Rio de Janeiro, abr. 2013. Disponível em: http://www.corecon-rj.org.br/jornal.asp. Acesso em: 27 maio 2013.

NADE/DRM - Equipe do Núcleo de Análise e Diagnóstico de Escorregamentos. Relatório Técnico sobre o Desastre de Petrópolis. Niterói, 2013. Disponível em: http://www.drm.rj.gov.br/ index.php/component/content/article/406. Acesso em: 27 maio 2013.

IBGE - Instituto Brasileiro de Geografia e Estatística. Censo Demográfico 2010 e Produto Interno Bruto dos Municípios. Disponíveis em: http://www.ibge.gov.br. Acesso em: 27 maio 2013.

IPCC. Climate Change 2007: Synthesis Report. Contribution of Working Groups I, II and III to the Fourth Assessment Report of the Intergovernmental Panel on Climate Change. IPCC, Genebra, 2007. Disponível em: http://www.ipcc.ch/. Acesso em: 26 maio 2013.

. Managing the Risks of Extreme Events and Disasters to Advance Climate Change Adaptation: A Special Report of Working Groups I and II of the Intergovernmental Panel on Climate Change. Cambridge University Press, Cambridge, 2012. Disponível em: http://www. ipcc.ch/. Acesso em: 26 maio 2013.

UNISDR. From Shared Risk to Shared Value: The Business Case for Disaster Risk Reduction. Global Assessment Report on Disaster Risk Reduction. United Nations Office for Disaster Risk Reduction (UNISDR), Geneva, 2013. Disponível em: http://www.preventionweb.net/english/hyogo/gar/2013. Acesso em: 27 maio 2013.

PNUD - Programa das Nações Unidas para o Desenvolvimento. Atlas do Desenvolvimento Humano no Brasil. 2003. Disponível em: http://www.pnud.org.br/ Acesso em: 27 maio 2013. 
